\title{
El Diálogo en el Aula con Estudiantes de Nivel Superior en Clases de Humanidades para Ingeniería
}

\author{
Sandra V. Ibarra ${ }^{1}$ y Alma A. Benítez ${ }^{2}$ \\ (1) Instituto Politécnico Nacional, Escuela Superior de Ingeniería Mecánica y Eléctrica, Unidad Zacatenco, \\ Lauro Aguirre No. 120 Col. Agricultura, Ciudad de México, México. (e-mail: sibarrag@ipn.mx) \\ (2) Instituto Politécnico Nacional, Centro de Estudios Científicos y Tecnológico 11 "Wilfrido Massieu", Centro \\ de Investigaciones Económicas, Administrativas y Sociales. Lauro Aguirre No. 120. Col. Agricultura, Ciudad \\ de México, México. (e-mail: abenitez@ipn.mx)
}

Recibido May. 14, 2018; Aceptado Jul. 17, 2018; Versión final Sep. 13, 2018, Publicado Feb. 2018

\begin{abstract}
Resumen
La presente investigación explora las enunciaciones entre el profesor y los estudiantes de Nivel Superior, durante la impartición de la unidad de aprendizaje de humanidades en la carrera de Ingeniería Eléctrica. El estudio tiene como objetivo explorar los actos de habla y el tipo de predominio de funciones del lenguaje para evidenciar la presencia del diálogo dentro del aula. La metodología es de corte cualitativo, llevado a cabo, a través de un cuestionario estructurado y de audiograbaciones que contribuyeron al análisis de la presencia o ausencia de diálogo dentro del aula. Los resultados obtenidos muestran que con base en las características de la enunciación no se logra el diálogo, y se confirma la presencia de la conversación con atributos de informalidad, cotidianidad y sentido lúdico de socialización.
\end{abstract}

\section{The Dialogue in the Classroom with Students of Higher Level in Humanities Classes for Engineering}

\begin{abstract}
This research explores communication between teacher and students of Higher-Level Education, during the teaching of the unit of Humanities for Electrical Engineering. The objective of the study is to examine the speech acts and the type of predominance of features of the language to demonstrate the presence of the dialogue within the classroom. The methodology is qualitative, carried out through a structured questionnaire and audio recordings that contributed to the analysis of the presence or the absence of dialogue within the classroom. The results show that, based on the characteristics of the communication, dialogue is not achieved, and the presence of the conversation is confirmed with attributes of informality, everyday life and playful sense of socialization.
\end{abstract}

Keywords: act of speech; language functions; dialogue 


\section{INTRODUCCIÓN}

La Escuela Superior de Ingeniería Mecánica y Eléctrica Unidad Zacatenco (ESIME Zacatenco) del Instituto Politécnico Nacional (IPN), a partir de la Reforma Académica Institucional para la actualización del Modelo Educativo y Académico de 2004, debe perfilarse hacia la necesidad de modificar la forma de impartir clases, "Centrado en el aprendizaje, dejando de lado la concepción tradicional del estudiante como un ente abstracto, mero receptor de conocimientos y de información" (IPN, 2004). Por lo cual se busca una nueva cultura del trabajo académico. En términos del IPN: "Este modelo deberá abarcar una visión institucional integral [...] incorporando [...] enfoques centrados en el aprendizaje, con una correcta adaptación de los sistemas formativos, basados en la introducción de metodologías de enseñanza que otorguen prioridad a la innovación, a la creatividad y al uso intensivo de las tecnologías de información y comunicación" (IPN, 2004, p.55)

Conforme a las exigencias impuestas por la sociedad actual hacia el conocimiento y los nuevos requerimientos en la competencia internacional, donde México ya está inscrito, se habla de la educación centrada en el aprendizaje y en los procesos de formación con predominio en las Tecnologías de la Información y la Comunicación. También se discute acerca de las modalidades de enseñanza no convencionales dentro de las aulas, asimismo, del actuar del profesor y los estudiantes a partir de la perspectiva enfocada en el aprendizaje. La forma de impartir clases es una visión que ha prevalecido por más de diez años, desde su implementación, por lo que resulta relevante hacer un alto en el camino y saber el estado que guarda; en específico, explorar los actos de habla y el tipo de predominio de funciones del lenguaje para evidenciar la presencia de la oralidad en el aula. Una observación para acercarnos a esta situación, es considerar a las humanidades como una asignatura e identificar los elementos que muestren los actos de habla y las funciones del lenguaje. De esta manera, se puede analizar el tipo de enunciaciones en el discurso dentro del aula para evidenciar la existencia o la ausencia del diálogo. En este sentido, se plantea la siguiente pregunta de investigación: ¿Qué características locutivas, ilocutivas, perlocutivas y funciones del lenguaje exhibe la oralidad durante los actos de habla entre el profesor y los estudiantes dentro del aula, en la unidad de aprendizaje de Humanidades?

El método dialógico, de acuerdo con McCrary et al. (2010) es retomado, hoy por hoy, en las diversas redes curriculares a nivel nacional y/o internacional, cuyo objetivo es, por una parte, la preparación de profesores y por otra, la fundamentación de cursos referentes a métodos dialógicos. Así, partiendo de las condiciones y los elementos que permiten comprender y analizar el diálogo dentro del aula, se requiere, de acuerdo con Velasco et al.(2008) "un marco de interacción comunicativa verdadera, guiada por pautas de equilibrio en la participación y la búsqueda colectiva del sentido, esto es, en el marco de un verdadero diálogo" (p.462), en esta dirección, Andreucci (2012) considera el aspecto central del diálogo, cuando el hablante y el oyente están en una interacción directa, y este último interpreta el mensaje del interlocutor. Sin embargo, dentro del aula el inconveniente surge cuando el profesor no se involucra en prácticas dialógicas y recurre a la reproducción de patrones con diálogos estereotipados.

El profesor habla acerca de una referencia teórica y de la realidad a partir de sus contenidos; tales referencias de la realidad pueden ser ajenas al estudiante en cuanto al contexto social y a la temporalidad que se le ha planteado del mismo, lo cual hace que el estudiante pierda el interés (Racionero et al., 2007). Asimismo, en este tipo de comunicación, de acuerdo con Ponce (2008), el discurso del profesor inicia desde su concepción del saber y el estudiante debe adquirirlo; este escenario da pauta para desencadenar múltiples dificultades en el proceso del diálogo, como lo menciona Velasco y Alonso: "Ciertamente, el educador sabe acerca del diálogo, más usualmente no lo practica. Saber (tener noticia) acerca de algo no garantiza que se le ponga en práctica; quizás simplemente que se le acepta: tener información no es tener conocimiento, para llegar a éste hay que creer y practicar, a fin de lograr experiencia [...] en la formación del educador no se le suele enseñar a dialogar ni suele emplearse el diálogo para su capacitación[...] de modo que mal se podrá pretender que el educador sea dialógico y la educación sea dialógica [...]," (Velasco et al., 2008, p. 468).

Se ha evidenciado que el diálogo no consiste en un simple envío de mensajes, más bien, adquiere conciencia acerca de lo que se dice, identificando la intención de compromiso y de responsabilidad en aprender con relación a los demás, consiguiendo un efecto transformador en los dialogantes, que al interpretar al otro -en una apreciación educativa- desarrolla su creatividad para lograr coincidir con el entendimiento, de acuerdo con su propio contenido, así como su capacidad para incorporar nuevos temas (Ponce, 2008). En esta situación emerge la importancia de analizar los actos de habla que manifiestan pensamientos, actitudes, sentimientos, intenciones e identificar en cuáles funciones del lenguaje recaen con mayor fuerza, para el alcance del aprendizaje, a fin de verificar si se logra el diálogo.

Es así como el modelo dialógica se presenta como una posibilidad en la cual el estudiante deja de ser espectador y expectante durante la clase, ante la explicación del profesor. En términos de Velasco: "[...] 
para pasar a hacer buen uso de las capacidades cognitivas [...] permitiéndosele cuestionar y someter a examen la información, discutirla con sus pares y construir conocimiento con ellos [...]" (Velasco et al., 2008, p. 462). En este sentido, se considera viable recurrir al diálogo, específicamente sobre temas de la unidad de aprendizaje de Humanidades, que requiere la interiorización en los contenidos para su comprensión y su aplicación.

El diálogo implica, en el escenario social del aula, delimitar el involucramiento y compromiso del estudiante y del profesor en un interés por y para el conocimiento de otro, pues reacciona ante la provocación discursiva del otro -estudiante o profesor- lo emplaza a identificar, analizar y movilizar (Andreucci, 2012), por su parte Skidmore et al. (2005), manifiestan que los estudiantes pueden conformar comunidades pequeñas relacionadas con alguna investigación, en este contexto surge la esencia de diálogo del discurso para conceder la construcción del conocimiento, proceso en el que confluyen las participaciones de cada uno de los miembros y ser examinadas críticamente en el discurso dentro del aula, evidenciando la complejidad del aprendizaje dialógico por su diversidad, ventajas y desafíos en dicho proceso. En este contexto es importante reconocer los indicadores para verificar la presencia o la ausencia del diálogo en el aula, razón por la cual se han considerado los siguientes atributos: dialogar con la otredad y tomar en serio los argumentos del otro. Así pues, se identifica el siguiente proceso; Proceso dialógico inferencial: Considera al otro en su enunciación. Anticipa e imagina las posibilidades de interpretación del otro, cooperando con su interlocutor en la construcción de una racionalidad; le permite negociar y colaborar de acuerdo a causalidades y formas de pensar plausibles para el otro (Velasco et al., 2008). Asimismo se rescata que las creencias o saberes previos de quien interpreta el diálogo son atribuidas al agente, pues por medio del conocimiento previo, se pueden entender las creencias nuevas.

Como consecuencia surge un espacio de creencias compartidas. El mismo autor considera que la interpretación o comprensión de la otra persona es un proceso en el que los involucrados adjudican sus propias creencias sobre el agente para comprenderlo, hacia una interpretación compartida (Ponce, 2008). Por otro lado, existen diferentes factores que actúan en la elaboración de interpretaciones de la realidad. Hablar de una realidad y producir otra. Referente externo (los problemas tematizados) pero se refiere a sí mismo. Interpretar para actuar en la realidad que se desea transformar. Condición del diálogo. Verse como ser inacabado que necesita ampliar sus creencias (Bastidas, et al., 2009). Si por sus características, el tipo de oralidad utilizado en el aula no es el diálogo, podría tratarse de una conversación y por ello no se concretan las reacciones de aprendizaje. La conversación no requiere del involucramiento con el otro, ni del compromiso de comprender lo que se dice, pues se propicia en ambientes cordiales con tónica informal; a menudo, radica en un encadenamiento de intercambios de opiniones producida por los agentes participantes que conversan de manera casual, mientras que el diálogo encadena intercambios en secuencias significativas (Alexander, 2008), por lo que no siempre se considera diálogo, dado que no es posible aprender, reflexionar o extraer comportamientos sólidos (Velasco et al., 2008). A consecuencia de ello, es posible revisar cómo se encuentra estructurada la interacción comunicativa oral entre profesor y estudiante a través del análisis de los actos de habla y las funciones del lenguaje, que permita la revisión de la estructura verbal en el aula, mediante los recursos referidos.

Al respecto, se recurre a la teoría de actos de habla de Austin (1962) por ser la pionera en visualizar al habla como una acción más allá de su función propia. Además aporta un modelo de categorías de los actos de habla (locucionario, ilocucionario y perlocucionario) que es pertinente y conveniente para esta investigación, y que servirá para interpretar las enunciaciones de acuerdo con su sentido y su referencia. Esta condición proporciona un revestimiento de intención, y si en la amplia gama de reacciones, el otro puede realizar una acción comunicativa que lo evidencie a través de actos perlocutivos. Por otra parte, Lozano (2010) considera que hablar una lengua consiste en realizar actos de habla; resultado de una emisión cuya intención se refleja en la fuerza ilocucionaria. Es una ventaja de tipo operativo para comprender la causa-efecto de la situación del aula. Ahora bien, el lenguaje y su forma es la plataforma para desarrollar el comportamiento humano, su práctica y su sentido. El comportamiento puede ser entendido como el contenido funcional del lenguaje (Ribes- Iñesta, 2007). En esta dirección se recurrió al aporte teórico de Jakobson (1984) para la revisión de los matices de las enunciaciones, el cual especifica las funciones del lenguaje inmersas en el proceso de comunicación, permitiendo determinar los atributos de los actos de habla del profesor hacia los estudiantes, visualizando los actos de habla como un mensaje. Tales funciones del lenguaje centran el análisis comunicativamente en las enunciaciones del mensaje, insertadas en el proceso comunicativo, no sólo en la interacción por sí misma.

Las funciones del lenguaje, de acuerdo con el autor, son las siguientes: Referencial, fundamentalmente de tipo informativo, está ubicada en el factor contextual. Emotiva, implica directamente al hablante que puede manifestarse con interjecciones y los matices emocionales. Conativa, ésta va directamente al oyente, con oraciones de tipo imperativo para hacer que actúe. De acuerdo con Waugh (1980), la función es por excelencia poética, sin embargo la creatividad del lenguaje se pueda presentar en contextos del diario vivir. 
Maybin et al., (2007) ofrecen como formas lingüísticas caracterizadas por repeticiones o figura literarias, pues atribuyen una presencia llamativa al mensaje, dotándolo de una apariencia atractiva.

La función fática incide en directo con el canal de comunicación, ya sea para abrir, cerrar o mantenerlo, con recursos como la pregunta temática, la poética en el mensaje, en el estilo o figuras para propiciar novedad en la expresión. Finalmente, la función metalingüística se encuentra en el lenguaje en cuanto al comprender el código utilizado, al aclarar conceptos y establecer definiciones en su uso.

\section{METODOLOGÍA}

La metodología que orienta la presente investigación es un estudio de corte cualitativo. La cual se realizó con un estudio de caso que permitió indagar la causa del problema planteado y con el análisis de las enunciaciones de los sujetos de acuerdo a los actos de habla y las funciones del lenguaje, establecer la dialogicidad en el aula. Se consideró oportuno -además de la verificación e identificación de las categorías teóricas de Austin y Jakobson, sobre el diálogo- la aplicación de un cuestionario estructurado para recabar la información complementaria, respecto de la aproximación hacia la comprensión del estudiante y del profesor, a fin de saber cómo se percibe la otredad a partir de la expresión oral y averiguar si hay instrucción para las participaciones y compromisos interpretativos hacia la unidad de aprendizaje. Las enunciaciones emitidas por el profesor y los estudiantes, durante la dinámica de clases, se registraron mediante la observación del discurso y la audiograbación de las mismas.

Se seleccionaron dos clases muestra en el aula para constituir el estudio de caso. Las enunciaciones entre el profesor y los estudiantes se obtuvieron a través de la audiograbación de dichas clases. Posteriormente, se realizó la transcripción, con el fin de ejecutar su análisis y su interpretación. Así mismo, se aplicó un cuestionario estructurado para obtener información complementaria del grupo estudiado. La clase se registró completamente, con una duración que fue desde los treinta minutos hasta una hora y media. Posteriormente hubo una selección de segmentos con las siguientes características: un segmento continúo y estable de actos de habla y de interacción comunicativa intensa entre ambos sujetos comunicantes sobre el tema de clase. Hay que hacer notar que, como posible sesgo, se contempló que al solicitar la autorización a los profesores para audiograbar, éstos se esmeraron en la impartición de la clase, sin embargo, no podían saber los aspectos en los cuales se enfocaría la investigación.

Las conversaciones grabadas permitieron analizar el objeto de estudio, abordando explicaciones interpretativas respecto de las variables de los actos de habla: intenciones, pensamientos, emociones, funciones del lenguaje (las categorías: referencial, emotiva, conativa, fática, poética y metalingüística), que se transmiten a través de los actos de habla utilizados en el diálogo en el aula. La delimitación de las dos clases muestra consistió en que cumplieran con la temática de la catedra correspondiente a la unidad de aprendizaje de humanidades II y el tema de humanismo impartido en los semestres $2^{\circ}$ y $3^{\circ}$ respectivamente, de la carrera de Ingeniería Eléctrica de la ESIME Unidad Zacatenco, en grupos formados hasta de 30 estudiantes. Para el perfil de los profesores se consideró lo siguiente: ser miembros de la Academia de Humanidades de Ingeniería Eléctrica, sexo masculino o femenino, con antigüedad en la práctica docente mayor de 8 años en la ESIME Zacatenco, activo frente a grupo, con posgrado y/o cursos de actualización docente en el IPN.

\section{ANÁLISIS Y DISCUSIÓN DE DATOS}

El análisis se realizó de acuerdo con la intervención del hablante, correlacionando la clasificación de actos de habla de Austin con las funciones del lenguaje de Roman Jakobson, para ello se diseñó un esquema de clasificación de datos, considerando las funciones de lenguaje y los actos de habla, en la tabla 1 se muestran las emisiones en el discurso en el aula.

Tabla 1. Emisiones en el discurso en el aula

\begin{tabular}{clc|c|c}
\hline & & \multicolumn{2}{c}{ Actos de habla } \\
\cline { 3 - 4 } Funciones del lenguaje: & Locución & llocución & Perlocución \\
\cline { 2 - 4 } 1. Emotiva & L-Emo & I-Emo & P-Emo \\
2. Referencial & L-Ref & I-Ref & P-Ref \\
3. Conativa & L-Con & I-Con & P-Con \\
4. Fática & L-Fat & I-Fat & P-Fat \\
5. Poética & L-Poe & I-Poe & P-Poe \\
6. Metalingüística & L.Met & I.Met & P.Met \\
& & & \\
\hline
\end{tabular}


Con base en la tabla 1 , se procedió a identificar la segmentación de actos de habla con las herramientas teóricas, cuya delimitación se realizó considerando la enunciación continúa, de acuerdo con la temática de la clase para utilizar los recursos de los actos de habla; con dinámica de interacción comunicativa constante que permitiera verificar las funciones del lenguaje y la retroalimentación entre ambas partes, con el fin de poder revisarla con el modelo de diálogo. Lo que permitió reconocer los tipos de enunciación (Funciones del lenguaje y los Actos de Habla) que son utilizadas por ambas clases de sujetos, así como la clasificación de los docentes, según el mayor o el menor empleo de algunos tipos.

La tabla 2 muestra los tipos de enunciaciones empleadas por ambas clases, reconociendo la segmentación identificada.

Tabla 2. Tipos de Enunciación

\begin{tabular}{|c|c|c|c|}
\hline \multicolumn{4}{|c|}{ Tipos de enunciación } \\
\hline & & Docente & Estudiantes \\
\hline \multirow[t]{4}{*}{$\begin{array}{l}\text { Clase } \\
1\end{array}$} & Segm. 1 & $\begin{array}{l}\text { L-Con } \\
\text { I-Fat } \\
\text { P-Fat } \\
\end{array}$ & $\begin{array}{l}\text { L-Ref } \\
\text { P-Ref }\end{array}$ \\
\hline & Segm. 2 & $\begin{array}{l}\text { L-Con } \\
\text { I-Ref } \\
\text { P-Fat }\end{array}$ & $\begin{array}{l}\text { L-Ref } \\
\text { P-Ref }\end{array}$ \\
\hline & Segm. 3 & $\begin{array}{c}\text { L-Con } \\
\text { I-Ref } \\
\text { P-Fat } \\
\end{array}$ & $\begin{array}{l}\text { L-Ref } \\
\text { P-Ref }\end{array}$ \\
\hline & Segm. 4 & $\begin{array}{c}\text { L-Fat } \\
\text { I-Ref-Met }\end{array}$ & P-Fat \\
\hline \multirow[t]{5}{*}{$\begin{array}{l}\text { Clase } \\
2\end{array}$} & Segm. 1 & $\begin{array}{l}\text { L-Ref } \\
\text { I-Con }\end{array}$ & P-Emo \\
\hline & Segm. 2 & $\begin{array}{l}\text { L-Ref } \\
\text { I-Con } \\
\text { P-Fat }\end{array}$ & $\begin{array}{l}\text { L-Ref } \\
\text { I-Ref }\end{array}$ \\
\hline & Segm. 3 & $\begin{array}{l}\text { L-Ref } \\
\text { I-Con } \\
\text { P-Fat }\end{array}$ & $\begin{array}{l}\text { L-Ref } \\
\text { I-Fat }\end{array}$ \\
\hline & Segm. 4 & $\begin{array}{l}\text { L-Ref } \\
\text { I-Emo } \\
\text { P-Fat } \\
\end{array}$ & P-Emo \\
\hline & Segm. 5 & $\begin{array}{l}\text { L-Ref } \\
\text { I-Ref } \\
\text { P-Fat } \\
\end{array}$ & P-Ref \\
\hline
\end{tabular}

Clase muestra 1: Consistió en una clase de repaso de contenidos con explicación y pregunta directa a los estudiantes sobre aspectos de los temas vistos anteriormente. A continuación, se aprecia parte de los actos de habla y funciones del lenguaje de la clase muestra 1 (Véase la tabla 2).

Profesor: - ¿cómo se informa usted?, ¿cuál es su medio de información para saber qué ocurre en el país?, ¿qué pasó ahorita en Reforma, en Iztapalapa o en el norte en Sinaloa?, ¿qué pasa en el país y en el mundo?, ¿por qué medios se informa usted?

Estudiante: - Yo por la radio o por periódico (Segm. 1)

Profesor: --La radio o periódico, ¿ventajas del periódico?

Estudiante: --Ah! Pues, porque en el periódico [pausa] este, nos informa de [pausa] este, temas de un lugar y pues como que sería más informativo para todas las personas. (Segm. 2)

Profesor: -Bueno, es una forma, a ver ¿qué más puede decir del periódico?

Estudiante: -Bueno, que por ejemplo lo que difiere al periódico de otras formas de comunicación masiva son [pausa] bueno, yo creo que más que nada la calidad, porque puede que la información sea más amplia digamos más este [pausa] en la en [...] por ejemplo en la televisión te da más información, pero quizá está manipulada, ¿no? Entonces, la información más fiable, yo creo que es el periódico, hay ciertos periódicos. (Segm. 3).

Profesor: - Confiable en un canal, teniendo un comunicador o en otro canal, según la emisora y según el comunicador también, en el periódico. ¿Cuál es la diferencia? Que mientras en la televisión tenemos una nota informativa, son por notas informativas que en media hora ipum! nos sueltan una cantidad de información, pero en pequeñas cápsulas informativas como le llaman. Mientras que en el periódico si elegimos un buen diario, vamos a tener el contexto de la información. ¿Qué es el contexto?

Estudiante: - ¿Lo que está escrito? (Segm. 4) 
En la enunciación de la profesora, el acto locucionario se evidencia cuando dice: cómo se informa usted: cuya función es conativa, de tipo imperativo, lo que inquirió la manera de informarse del estudiante (acto ilocucionario), permitiendo el acto perlocutorio cuando instó a que dijera los medios por lo que se informa el estudiante, para mantener el canal de comunicación abierto con el estudiante y continuar informando con los contenidos anteriores, es decir se impulsa la función fática. La función más empleada por el estudiante al realizar sus respuestas es la referencial, ubicando los actos de habla con base a los cuestionamientos (Como se advierte en los segms. 1 y 2 , respectivamente).

En el segmento 3 (Véase segm. 3) se aprecia el acto locutivo, cuando dice: diga más del periódico, cuya función de lenguaje es conativa de tipo exhortativa de mandato, para proceder con el acto ilocutivo, pues incitó la respuesta sobre más información que tenga sobre el periódico, utilizando la función referencial sobre el contenido informativo y la función fática para mantener contacto comunicativo, pues obliga a generar una respuesta con el acto perlocutivo. Respecto a la función por el estudiante es referencial, ya que ubica los actos de habla, debido al cuestionamiento emitido por la profesora.

La profesora emplea los actos ilocucionarios, es decir, el significado que tiene el acto locucionario, los cuales muestran ausencia de una estructura verbal, calculada con la intención de a alcanzar una meta, los actos de habla son de tipo fático y referencial, permitiendo mantener abierto el canal de comunicación para continuar informando en la clase, pero destacan los momentos en que se desaprovechan las posibilidades para generar actos perlocucionarios, perdiendo la posibilidad de actos de habla en el estudiante. Aunque se recurre, al final de la enunciación, a la función metalingüística, con actos perlocutorios para retroalimentar a los sujetos comunicantes y propiciar la función fática en el estudiante, manteniendo la apertura en la comunicación (Como se aprecia en el Segm. 4).

De acuerdo con las preguntas y las enunciaciones explicativas del tema por parte de la profesora, no proporciona regulación a través de actos locucionarios para establecer una mecánica de intervenciones sobre la materia. La consecuencia es la falta de pautas que orienten la forma de abordar el tema. La enunciación muestra que la manera de retribuir el reconocimiento a los estudiantes por sus comentarios, fue cuando éstos coincidían con la respuesta esperada por la profesora. En ese sentido, la búsqueda de aprobación se vuelve más importante. Los enunciados son extensos, prevaleciendo la redundancia y la retiración de la idea en la búsqueda de mantener la comunicación activa, pero al hacerlo, se induce la respuesta, por lo que se pierde la intención de verificar el contenido expuesto por el estudiante y poder identificar la intención ilocutiva de escuchar respuestas acertadas, predeterminadas o esperadas.

Las funciones predominantes del lenguaje identificadas en la profesora fueron las siguientes: primeramente, la fática, que consiste en mantener activo el contacto entre los sujetos comunicantes. Después, la referencial, por encontrarse contenido de tipo informativo, básico en las respuestas de los estudiantes que buscan coincidencia con la profesora. Luego, la función conativa, misma que es de tipo imperativo o exhortativo orienta a la enunciación de tal forma que obliga al estudiante a hablar, dando respuestas forzadas, que coinciden con la orientación de la temática de la profesora. La función referencial se identificó como la más utilizada en las respuestas del estudiante, dado que ubica los actos de habla debido a los cuestionamientos; este es su referente más inmediato para emitir su contestación. La función referencial es informativa en relación con lo que se esté hablando, por lo que, al ser expresiones sobre el tema con contenido básico -simples en su estructura o expresadas con vaguedad-permiten considerarse en un nivel informativo escaso. Se observa que se recurrió a la función emotiva en una expresión admirativa afirmativa; se utilizó como motivador entusiasta por coincidir la idea del estudiante con la idea predeterminada buscada por el profesor.

Clase muestra 2. Se caracterizó por utilizar una lectura de referencia como material que el profesor proporcionó previamente a los estudiantes, para ser comentado en clase. Siendo este texto el referente principal para emitir las enunciaciones, aunque también se recurre a información dada en clases anteriores. A continuación, se aprecia parte de los actos de habla y funciones del lenguaje de esta clase muestra (Véase el tabla 2).

Profesor: - ¡Híjole! aquí en México, como que va a tardar un poquito más.

Estudiantes (todos). [risas] (Segm. 1)

Profesor: - No sé, no sé, no, ya, en México como que [pausa]. A ver, si yo les pregunto, un modelo de ser humano, ¿a quién me pondrían?

Estudiantes (E3): - Morelos (Segm. 2)

Profesor: - A ver, Denisse dice: no hay. A ver [pausa] quién, algún mexicano que podría decir: este actualmente podría ser el modelo a aspirar para ser humano ¿quién sería ahorita?

Estudiante: - ¿humano? (Segm. 3)

Profesor: - A ver, ahorita, ¿quién? ¿Chespirito? 
Estudiante: - Chabelo [risas] (Segm. 4)

Profesor: - Bueno, San Francisco ya le dio la vuelta al mundo. ¿Algún mexicano?

Estudiante: - Psss, pero no necesariamente tiene que ser, no tiene que ser, no tiene que tomar, ora sí, un [pausa] o sea, no ocupa un alto nivel en la sociedad, sino que una persona puede ser tan ser humano, pero la verdad, no lo conocemos, puede ser como usted dice, tan humilde que lo podemos encontrar en la calle sin darnos cuenta, puede ser la persona más humana en México, puede ser un vago, no sé, un policía, un pobre. (Segm. 5).

Los actos ilocutivos del profesor están direccionados a obtener un acto perlocutivo inmediato, es decir, concretado en acciones inmediatas logradas con la ilocución, la función conativa mantiene un orden y regulación para el actuar del estudiante ante la instrucción imperativa del profesor, por lo cual, delimita y centra las acciones del estudiante debido a la dinámica de clase. La función conativa se ve suavizada con la diversidad de manifestaciones de la función emotiva para destensar las enunciaciones imperativas del profesor (Segm. 1).

El profesor, al dirigirse hacia los estudiantes, utiliza el acto perlocutivo para proponer personajes públicos. La función del lenguaje es de tipo fático, manteniendo el canal comunicativo. Al formular un nombre como modelo de ser humano, el estudiante realiza un acto perlocutivo, logrando una respuesta con expresión emotiva (Segm. 4).

Cuando el profesor establece el acto ilocutivo para mencionar a otro mexicano y propiciar la reflexión en la relación de datos proporcionados, dejando al final la enunciación incompleta, a modo de pregunta, con la intención de ser completada por el estudiante, quien produce el acto perlocutorio, cuya función del lenguaje es referencial, pues argumenta que, de acuerdo con lo explicado por el profesor, puede ser alguien humilde (Segm. 5).

En esta dinámica de clase, los actos ilocutivos del profesor están direccionados a obtener actos perlocutivos inmediatos del estudiante, basados en el reforzamiento de conceptos vistos anteriormente en clases o del texto abordado en ese momento. Es así como el estudiante completa las ideas del profesor en actos perlocutivos, aportando conceptos sin ser propiciados por los actos ilocutivos del profesor, mismos que el docente integra en su siguiente enunciación.

Las expresiones del profesor mostraron mayor presencia en la función referencial o informativa, así como en la fática para mantener abierto el canal de comunicación. Les siguen la función emotiva, al identificar el uso de la exclamación, lo festivo o lo jocoso, integrando a los estudiantes para destensar la emisión conativa, dar órdenes y regular el actuar, por lo que fueron utilizadas para equilibrar las intenciones entre sí. De menor uso, apareció la función metalingüística, al aclarar el significado de palabras, al igual que la poética, con la inclusión de vocablos nuevos, atractivos al ser expresiones poco comunes; sin embargo, al ser utilizadas contribuyeron a producir reacciones de actos de habla en los estudiantes.

Se identificó que sin la lectura, los actos de habla de los estudiantes son escasos y vacilantes, como sucede en las enunciaciones al buscar a un personaje con atributos humanistas en el México actual (Véanse segms. 2 y 3) de modo que, la función es de tipo referencial, basada en personajes públicos de la televisión.

El profesor también recurre a repetir las palabras finales con las que completa la idea de la enunciación. Esta reiteración produce actos de habla perlocutivo con funciones de tipo fático para buscar el contacto constante con el estudiante, vinculados con la información de la lectura o de la clase previa. La estructura verbal del mensaje es predominantemente referencial, centrada en el contexto de la clase.

En este contexto, el profesor logra el primer acercamiento para establecer el vínculo de ideas, con conceptos de la lectura y de las clases previas, relacionando los nuevos saberes para comprender al profesor. Al ser conocimientos compartidos, abren paso a la siguiente aproximación: la búsqueda de la interpretación compartida, pero se ve truncada al no haber preguntas por parte del estudiante, en su exploración para encontrar coincidencia con el profesor, y por ser poco utilizada la función metalingüística, ubicada en la retroalimentación.

\section{DIÁLOGO EN EL AULA}

A partir del carácter dialógico se identificaron los siguientes indicadores de diálogo para verificar la existencia o su carencia en las clases muestra. Indicador 1: Dialogar con la otredad. Indicador 2: Proceso dialógico inferencial. Indicador 3: Creencias o saberes., Indicador 4: Consecuencia del diálogo., Indicador 5: Interpretación o comprensión de la otra persona., Indicador 6: Hablar de una realidad y producir otra.; Indicador 7: Condición del diálogo. En la tabla 3 se expone la presencia de diálogo en el intercambio didáctico: 
Tabla 3. Presencia de diálogo en las clases muestra

Presencia de indicadores de diálogo en el intercambio didáctico

Indicador 1 Indicador 2 Indicador 3 Indicador 4 Indicador 5 Indicador 6 Indicador 7 Clase 1

NO

Clase 2

NO

Clase Muestra 1. El estudiante emite respuestas carentes de argumentación, por lo que no alcanza un nivel de igualdad entre los sujetos comunicantes durante la relación establecida (Carencia de Indicadores 1 y 2 ). La simpleza o vaguedad de su contenido no proporciona un carácter de seriedad, en el sentido de aportación de datos y ser reconocido por el otro y a la vez interesarse en sus respuestas, ampliando sus saberes (Carencia de Indicador 3).

La profesora orienta sus actos de habla para obtener respuestas predeterminadas establecidas en clases anteriores. El estudiante al no externar sus saberes previos, disminuye la interacción entre los sujetos comunicantes, evitando la posibilidad de fortalecer nuevos conocimientos; al no emitir sus saberes, revela la falta de conocimientos previos sobre el tema (Carencia de Indicadores 3 y 5 ).

Lo anterior, también es indicador de la ausencia para tomar con seriedad los argumentos del otro (Indicador 1), por quien en ese sentido no se interesa en su enunciación. Situación que puede atribuirse a los referentes externos utilizados como ejemplos para los temas de clase, por no ser propuestos por los estudiantes, o bien por la falta de saberes para hacerlo (Indicador 3). Por lo que se advierte la ausencia de diálogo, pues la interacción comunicativa es limitada o nula.

Clase Muestra 2. El orden de los actos de habla, tanto del profesor como de los estudiantes, permite considerar algunos argumentos del otro, tomando como referencia la lectura anticipada, sin embargo, no alcanza la interpretación. Se puede considerar como una coincidencia, pues no se establecen preguntas. (Indicador 1. Carencia de Indicador 5).

Los estudiantes participan con el profesor para concluir las enunciaciones, las cuales están basadas en el contenido de la lectura. Al completar la enunciación, repiten conceptos, pero no logran expresar actos de habla para verificar la interpretación del otro, por lo que no se logra la negociación de causalidades ni la identificación de formas de pensar admisibles del otro (Carencia de Indicador 2).

Leer y recurrir a conceptos e ideas de clases previas permiten vincular el entendimiento entre los sujetos comunicantes (Indicador 3). Este vínculo permite un primer acercamiento del proceso de diálogo, por tratarse de saberes o creencias compartidas (Indicador 4). Es una plataforma para iniciar la búsqueda de una interpretación compartida, no obstante, se limita el proceso por la falta de preguntas por parte del estudiante para establecer puntos en común con el profesor, en consecuencia, la coherencia con los saberes del profesor, que den origen a la construcción de nuevas hipótesis al contrastar sus criterios con las respuestas del profesor (Carencia de Indicador 5).

El empleo de preguntas por parte del profesor otorga al estudiante la reflexión del contenido teórico en el contexto de la clase cuando se transfiere a la realidad los contenidos referenciados por el profesor, así como la búsqueda por parte del estudiante a través de ejemplos con relación a su realidad inmediata, aunque el referente le es difícil de identificar mostrando la falta de similitud con el profesor y ubicar con mayor facilidad los referentes de su realidad (Carencia Indicadores 6 y 7 ).

Con base en la exposición, se valora la presencia de algunos indicadores (1, 3 y 4), donde el profesor induce las respuestas de sus estudiantes, pues revela el deseo de creer que su estudiante aprendió, esto es, cuando logra que el alumno repita la información vista en clase, no consiguiendo la etapa de negociación de causalidades, ni de formas de pensar del otro. Los sujetos comunicantes no se perciben en igualdad para expresarse, impidiendo el reconocimiento del otro con seriedad e interesarse en sus respuestas y en una ampliación de saberes. Por lo anterior, pese a los esfuerzos del profesor en tratar de mantener la comunicación, empleando diversas interrogantes al estudiante, las intervenciones de habla no logran las primeras fases del modelo de diálogo, y sólo permanece la sucesión de intervenciones en el plano de la conversación, pues las participaciones no presentan trascendencia hacia la comprensión y la ampliación de sus saberes, incorporando la del otro. 


\section{RESULTADOS}

Finalmente, del análisis de la teoría de los actos de habla, las funciones del lenguaje y el modelo de diálogo se presentan los siguientes resultados a partir del contexto en el aula. La formación académica del profesor no determina la creación de actos de habla planeados en la búsqueda del diálogo para el aprendizaje, aunque puede obedecer a un estilo personal de socialización. La función del lenguaje predominantemente es la informativa o función referencial seguida de la fática, por tratarse de explicaciones largas informativas, pero puede carecer de dirección al aprendizaje en los actos ilocutivos, como lo menciona Giraldo et al., (2009), quienes llevaron a cabo la medición comunicativa entre catorce docentes; la experiencia mostró una comunicación de tipo expositiva para dar a conocer al estudiante la tarea a desarrollar. El profesor presenta contenido, pero no se concreta en lograr una reacción perlocutiva en el estudiante. Muestra interacción comunicativa, pero no implica diálogo, hay alternancia de participación pero sin garantizar actos perlocutivos. Las respuestas cortas con poca contribución informativa corresponden a preguntas conativas exhortativas de diseño cerrado, que caen en lo inductivo, lo cual no contribuye a la construcción de ningún conocimiento.

Los conceptos previos, mediante lecturas y datos de las clases, contribuyen para estructurar actos ilocutivos con un sentido y una referencia evidentes con el objetivo de generar actos perlocutivos en beneficio del aprendizaje. Se conserva activo el proceso comunicativo, sin embargo, la ausencia de la vinculación de saberes previos y nuevos, así mismo de la lectura previa como material de referencia, proporciona la interpretación de contenido de los sujetos como saberes previos. Las extensas exposiciones originan falta de actividad en los estudiantes para realizar perlocuciones, no obstante, incorporar estrategias como mejorar la frase, permiten generar actos perlocutivos y la afirmación del otro dentro de la dialogicidad, implementando la apertura a este tipo de oralidad. La función emotiva se observó en el profesor para producir la coincidencia del estudiante con la idea del docente, con carácter inductivo. Los resultados obtenidos reflejan que ante la evidencia de la enunciación en contextos de cotidianidad, la falta de planeación es un factor concluyente para lograr una determinada consecuencia en el estudiante. Lo cual permite evidenciar la falta de impacto para emprender el primer acercamiento expuesto en el modelo de diálogo. Con base en el análisis y por las diversas propiedades de las enunciaciones del profesor, con predominio cuantitativo, el estudiante continua siendo un espectador y por más variedad del estilo de habla del profesor, se sigue presentando centrada en la enseñanza, dentro de los parámetros de un escenario conversacional.

El análisis de las clases-muestra identificó dos formas habituales en la dinámica de la asignatura de humanidades por parte del profesor: La primera, como una explicación amplia con cuestionamiento directo, explicación con parafraseo, concluyendo frases y lectura. La segunda, como una explicación expositiva inicial con sesión final de dudas y práctica. Estas variantes evidenciaron que, aunque son diferentes en las técnicas didácticas empleadas para que el estudiante adquiera el contenido, se hallan limitadas por la escasa planeación del diseño de las expresiones verbales. Pues, por más esfuerzo que efectúe el profesor para favorecer las explicaciones voluntarias en el estudiante, estas son escasas, de básica participación o contenido pobre para contribuir al conocimiento del otro. Después de evidenciar la ausencia de dialogicidad para la incorporación de nuevos conocimientos, se distingue que la clase de oralidad que se constituyó es la conversación, debido a su estilo informal, sin planeación y compuesta por intervenciones de los estudiantes con emisiones monologales. En consecuencia, no se logra concretar nuevos saberes, es decir, se mantiene el estilo sin llegar al diálogo, situación que hace indudable el predominio de la función fática o la relativa a conservar contacto comunicativo, en sentido socializador, así como su carácter informal, coloquial y cotidiano, perdiendo la posibilidad de aprovechar las cualidades de la comunicación educativa.

\section{CONCLUSIONES}

De acuerdo a los resultados de este estudio, y de su discusión y análisis se pueden extraer las siguientes conclusiones principales:

1) las enunciaciones extensas del profesor revelan los actos ilocucionarios, debido a la ausencia de una estructura verbal orientada a una meta o finalidad, cuya función es de tipo referencial al ser expresiones del tema básico para buscar cuestionamiento e inducir las respuestas. En consecuencia se pierde la intención de verificar el contenido del estudiante respecto a las clases para escuchar respuestas predeterminadas (acto ilocutivo), cuya función conativa orienta la enunciación de modo que obliga al estudiante a hablar, dando respuestas forzadas que resultan ser coincidentes con la temática del profesor;

2) el profesor en la enunciación presenta actos de habla de tipo fático y referencial para mantener abierta la comunicación, concretándose sólo en la ilocución hacia la perlocución, debido a la explicación continúa sin espacios para reflexionar, en consecuencia, generar baja actividad en el estudiante con funciones de tipo 
referencial, los actos ilocutivos del profesor (clase muestra 2) están direccionados a obtener actos perlocutivos inmediatos, el estudiante completa la idea del profesor en actos perlocutivos, aportando posturas sin ser propiciadas por los actos ilocutivos del profesor, dichos actos se realizan a través de las funciones referencial y fática;

3) la presencia del predominio de la función fática en sentido socializador y la función referencial de tipo informativo limitan los actos de habla para generar reacción perlocutiva en los estudiantes, por lo que no hay ampliación de saberes, sino sólo a nivel informativo. Se concluye que el tipo de oralidad establecido es la conversación, por su estilo informal, cotidiano, lúdico sin planeación y compuesto por intervenciones de los sujetos comunicantes con emisiones monologales, motivo por el cual no se concretan los nuevos conocimientos en el estudiante, por lo que no se logra el diálogo (Velasco y Alonso, 2008) durante la dinámica de actos de habla en el aula; y

4) finalmente, se advierte la necesidad de planificar estrategias didácticas para motivar el diálogo, generando en el estudiante actos perlocutivos como retroalimentación, es decir, expresiones involucradas en el proceso de aprendizaje, producto de la intención de los actos ilocutivos emitidos por el profesor, el cual deberá enfocarse en aspectos específicos de la temática de clase.

\section{AGRADECIMIENTOS}

Las autoras agradecemos el apoyo otorgado por el Instituto Politécnico Nacional para realizar y presentar este artículo de investigación, cuyo número de registro es el 20150156.

\section{REFERENCIAS}

Alexander, R.J., Culture, dialogue and learning: Notes on an emerging pedagogy, $10^{\text {th }}$ International Conference Education, Culture and Cognition: intervening for growth, 91-114, Durham, UK, 10-14 de Julio (2008)

Andreucci, P., El talento: una construcción en y desde la pedagogía dialógica, ISSN: 0717-7798, Rev. Psicoperspectivas, Individuo y Sociedad 11(2), 185-205 (2012)

Austin, J., How to do things with words, $1^{\circ}$ Ed., London, Great Britain (1962)

Bastidas, M., F. Pérez y otros cuatro autores, El diálogo de saberes como posición humana frente al otro: referente ontológico y pedagógico en la educación para la salud, ISSN: 0120-5307, Investigación y Educación en Enfermería, 27(1), 104-111 (2012)

Giraldo, L.L., E.O. Rubio y J.O. Fernández, Caracterización del discurso pedagógico del docente de Educación Física e identificación de los actos de habla que estimulan la creatividad motriz, ISSN: 1578-2174, Rev. Ágora para la duración física y le deporte, 11(1), 25-41 (2009)

IPN, Materiales para la Reforma. Un Nuevo Modelo Educativo para el IPN. 1a Ed., 51-55, Instituto Politécnico Nacional, Distrito Federal, México (2003)

Jakobson, R., On linguistic aspects of translation, $2^{\circ}$ Ed., 38-43, Routledge, Routledge, Londres, Inglaterra (2004)

Lozano, E., La interpretación y los actos de habla, ISSN: 2011-799X, Mutatis Mutandis: Revista Latinoamericana de Traducción, 3(2), 333-348 (2010)

Maybin, J. y J. Swann, Everyday Creativity in Language: Textuality, Contextuality, and Critique, doi: 10.1093/applin/amm036, Applied Linguistics, 28(4), 497-517 (2007)

McCrary, N. y J. M. Mazur, Conceptualizing a narrative simulation to promote dialogic reflection: Using a multiple outcome design to engage teacher mentors, Educational Technology Research and Development, 58(1), 325-349 (2010)

Ponce, B.H., El diálogo en la interpretación del otro, ISSN: 1560-6139, Rev. Persona, 11(1), 69-74 (2008)

Ribes-Iñesta, E., El Lenguaje, Aprendizaje y Conocimiento, ISSN: 0185-6073, Revista Mexicana de Psicología, 24(1), 714 (2007)

Racionero, S. y R., Valls, Dialogic Learning: A communicative approach to teaching and learning; in The Prager Handbook of Education and Psychology, Prager, 548-557 Westport, Connectiutd, London (2007)

Skidmore, D. y D. Gallagher, A Dialogical Pedagogy for Inclusive Education; Inclusive and Supportive Education Congress, 23-29, Glasgow, UK United Kingdom, 1-4 de Agosto (2005)

Velasco, J. A. y L. A. González, Sobre la Teoría de la Educación Dialógica, ISSN: 1316-4910, Rev. Venezolana de Educación: Educere, 12(42), 461-470 (2008)

Waugh, L., The Poetic Function in the Theory of Roman Jakobson, doi: 10.2307/17772352, Poetics today, 2(19), 57-82 (1980) 